\title{
Hematuria en la niñez: revisión sistemática cualitativa
}

\author{
Hematuria in the childhood \\ A systematic cualitative review
}

John J. Paz-Montañez MD', Yolanda Mueses-Guerrero MD2, Jaime M. Restrepo-Restrepo MD', Roberth A. Ortíz-Martínez MSc ${ }^{4}$, María A. Acosta-Aragón PhD

Introducción: la hematuria aislada en niños es un hallazgo frecuente en la práctica clínica diaria del médico general y el pediatra, y un reto diagnóstico para averiguar su etiología y manejo. Objetivo: describir las pautas básicas para el diagnóstico y manejo de la hematuria aislada en menores de 18 años por el médico general y el pediatra. Materiales y métodos: se realizó una búsqueda bibliográfica sistemática en las bases de datos PubMed, ScienceDirect, LILACS y Embase, utilizando palabras claves del DeCS (español) y MeSH (inglés), mediante las combinaciones con la conjunción "AND» o la disyunción "OR», de manuscritos tipo estudios observacionales, ensayos clínicos, guías de práctica clínica y revisiones sistemáticas, publicados entre 1995 y 2017, que describieran el diagnóstico y el manejo básico de la hematuria. La calidad de los artículos fue evaluada por medio de los instrumentos PRISMA, CONSORT y STROBE, según correspondiera. Resultados: se identificaron 402 publicaciones, de las cuales 34 cumplieron con los criterios y 28 fueron seleccionadas para realizar esta revisión. Conclusiones: la hematuria aislada se define por el hallazgo de cinco eritrocitos por campo de alto poder en orina fresca centrifugada o más de cinco eritrocitos por microlitro en orina fresca no centrifugada. El diagnóstico y tratamiento se realiza por pasos: a) historia clínica y b) confirmación por microscopía óptica de alta resolución. Diferenciar la hematuria benigna de las que requieren paraclínicos de extensión es primordial para confirmarla más tempranamente, evitar procedimientos invasivos, disminuir el gasto en salud y hacer un seguimiento predictivo.

Palabras clave: hematuria, niños, diagnóstico, revisión sistemática.

Paz-Montañez JJ, Mueses-Guerrero Y, Restrepo-Restrepo JM, Ortíz-Martínez RA, Acosta-Aragón MA. Hematuria en la niñez: revisión sistemática cualitativa. Medicina \& Laboratorio 2017; 23: 351-364.

\footnotetext{
${ }^{1}$ Médico residente, programa de Pediatría, Facultad de Ciencias de la Salud, Universidad del Cauca. Popayán, Colombia. Correo electrónico: jpazm@unicauca.edu.co.

2Médico residente, programa de Pediatría, Facultad de Ciencias de la Salud, Universidad del Cauca. Popayán, Colombia.

${ }^{3}$ Médico, Nefrólogo Pediatra, Fundación Valle de Lili, Centros Renales Hermanos TRIO de la Sociedad Internacional de Nefrología (ISN). Cali, Colombia.

${ }^{4}$ Médico, especialista en Ginecología y Obstetricia, Universidad del Cauca, Magíster en Epidemiología, Universidad del Valle. Cali, Colombia.

${ }^{5}$ Médica y Cirujana, MSc en Biología, área Genética Clínica, PhD en Genética de Poblaciones Humanas y Genética Forense. Profesora titular, Departamento de Pediatría, Facultad de Ciencias de la Salud, Universidad del Cauca. Popayán, Colombia. Conflicto de intereses: los autores declaran que no tienen conflicto de intereses Medicina \& Laboratorio 2017; 23: 351-364

Módulo 13 (Química Clínica), número 15. Editora Médica Colombiana S.A. $2017^{\circ}$ Recibido el 18 de julio de 2017; aceptado el 25 de agosto de 2017
} 
a presencia de sangre en la orina de un

niño, sea macro o microscópica, es un motivo de consulta frecuente que ocasiona gran ansiedad en el niño y en la familia por la percepción de ser una enfermedad grave [1]. La hematuria es la alteración urinaria más común en la infancia, con una prevalencia aproximada en niños y niñas de diferentes países entre los seis y 15 años, del $1 \%$ al $2 \%[2,3]$. En niños la hematuria más frecuente es la microscópica con un $60 \%$ al $70 \%$ de los casos, mientras que la macroscópica tiene una prevalencia de al menos 1,3 casos por cada 1.000 niños, donde las etiologías no traumáticas más frecuentes son la nefropatía por inmunoglobulina A (IgA) y la glomerulonefritis posestreptocócica [4].

Los resultados del tamizaje masivo se han utilizado como marcadores de lesión renal y, en los últimos años, han dado paso al análisis de los grupos de riesgo (p. ej. con anormalidades congénitas, infecciones sistémicas, diabetes, sobrepeso, entre otros), en los cuales la hematuria se ha convertido en una herramienta para el diagnóstico de glomerulopatías, tubulopatías o uropatías, susceptibles de tratamiento temprano, como se ha demostrado en otros estudios [5].

El incremento en la frecuencia de la insuficiencia renal crónica ha llevado a un aumento exponencial de la carga económica de la mayoría de los presupuestos en salud en el mundo, por lo que toda actividad tendiente a disminuirla es un objetivo principal en la práctica de la prevención primaria en la nefrología. Se calcula que en el 2004, en Colombia, las diálisis alcanzaron un costo de $\$ 450.000 .000 .000$, y que en los últimos años consumieron alrededor del $2 \%$ y el $4 \%$ del gasto en salud del país y de la seguridad social en salud, respectivamente. En cuanto a los trasplantes renales se invirtieron
$\$ 37.500 .000 .000$ para el año 2009 [6]. Todo lo anterior, indica que se requiere obtener un diagnóstico precoz de la enfermedad, como lo puede brindar la presencia de hematuria al inicio de una enfermedad glomerural o renal.

El objetivo de este manuscrito es describir las pautas básicas de diagnóstico y manejo de la hematuria aislada en menores de 18 años para el médico general y para el pediatra en atención primaria, con sus principales actualizaciones.

\section{Materiales y métodos}

Se realizó una búsqueda bibliográfica sistemática teniendo en cuenta las palabras claves consignadas en los Descriptores en Ciencias de la Salud (DeCS) en español y los Encabezados de Temas Médicos (MeSH; del inglés, Medical Subject Headings) en inglés en las bases de datos PubMed, ScienceDirect, LILACS y Embase, y utilizando las combinaciones con la conjunción "AND» o la disyunción "OR», de manuscritos publicados entre 1995 y 2017, que describieran el diagnóstico y el manejo básico de la hematuria en menores de 18 años.

Como criterios de inclusión se tomaron estudios observacionales, ensayos clínicos, guías de práctica clínica y revisiones sistemáticas. La calidad de los artículos fue evaluada por medio de los instrumentos PRISMA (del inglés, Preferred Reporting Items for Systematic reviews and Meta-Analyses) para las revisiónes sistemáticas, CONSORT (del inglés, CONsolidated Standards Of Reporting Trials) para los ensayos clínicos y STROBE (del inglés, STrengthening the Reporting of OBservational studies in Epidemiology) para los estudios observacionales. 


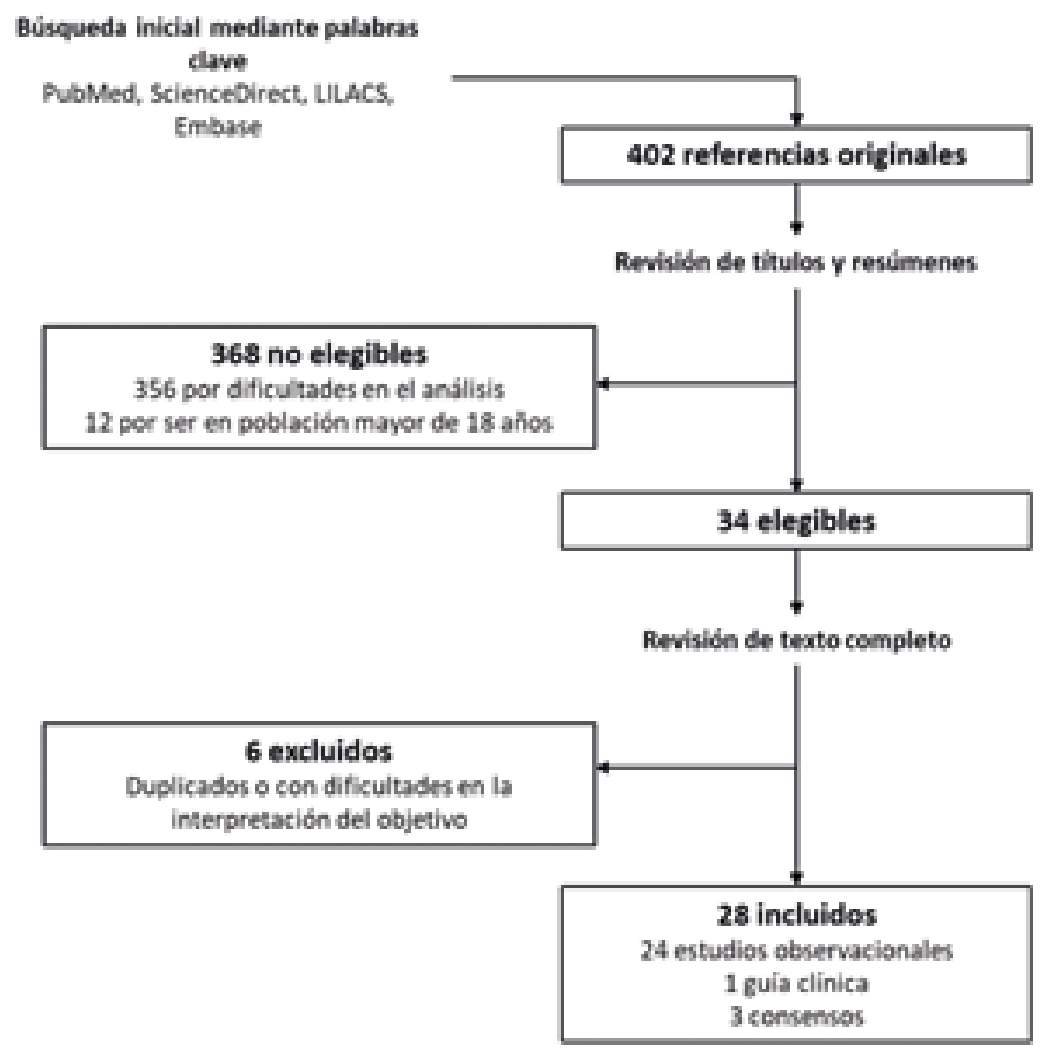

Figura 1. Diagrama de flujo de la revisión sistemática cualitativa sobre el diagnóstico y el manejo básico de la hematuria en menores de 18 años.

\section{Resultados}

La búsqueda sistemática, mediante las palabras clave indicadas, arrojó un total de 402 publicaciones, de las cuales 356 se descartaron por dificultades de análisis y 12 por incluir población adulta mayor de 18 años. Posteriormente, seis artículos se retiraron por ser referencias repetidas 0 por dificultades en la interpretación del objetivo de los mismos. Finalmente, se seleccionaron 28 publicaciones, de las cuales 24 fueron estudios observacionales, una correspondía a una guía de práctica clínica $y$ tres consensos de expertos que fueron incluidos a criterio de los autores (véase figura 1 y tabla 1), las cuales fueron consideradas de transcendencia para describir el diagnóstico y el manejo básico de la hematuria en menores de 18 años.

\section{Discusión}

\section{Definición de la hematuria}

Puede variar según los autores, pero las que tienen más aceptación son $[7,8]$ :

Hematuria microscópica aislada: cuando existen más de cinco eritrocitos por campo 


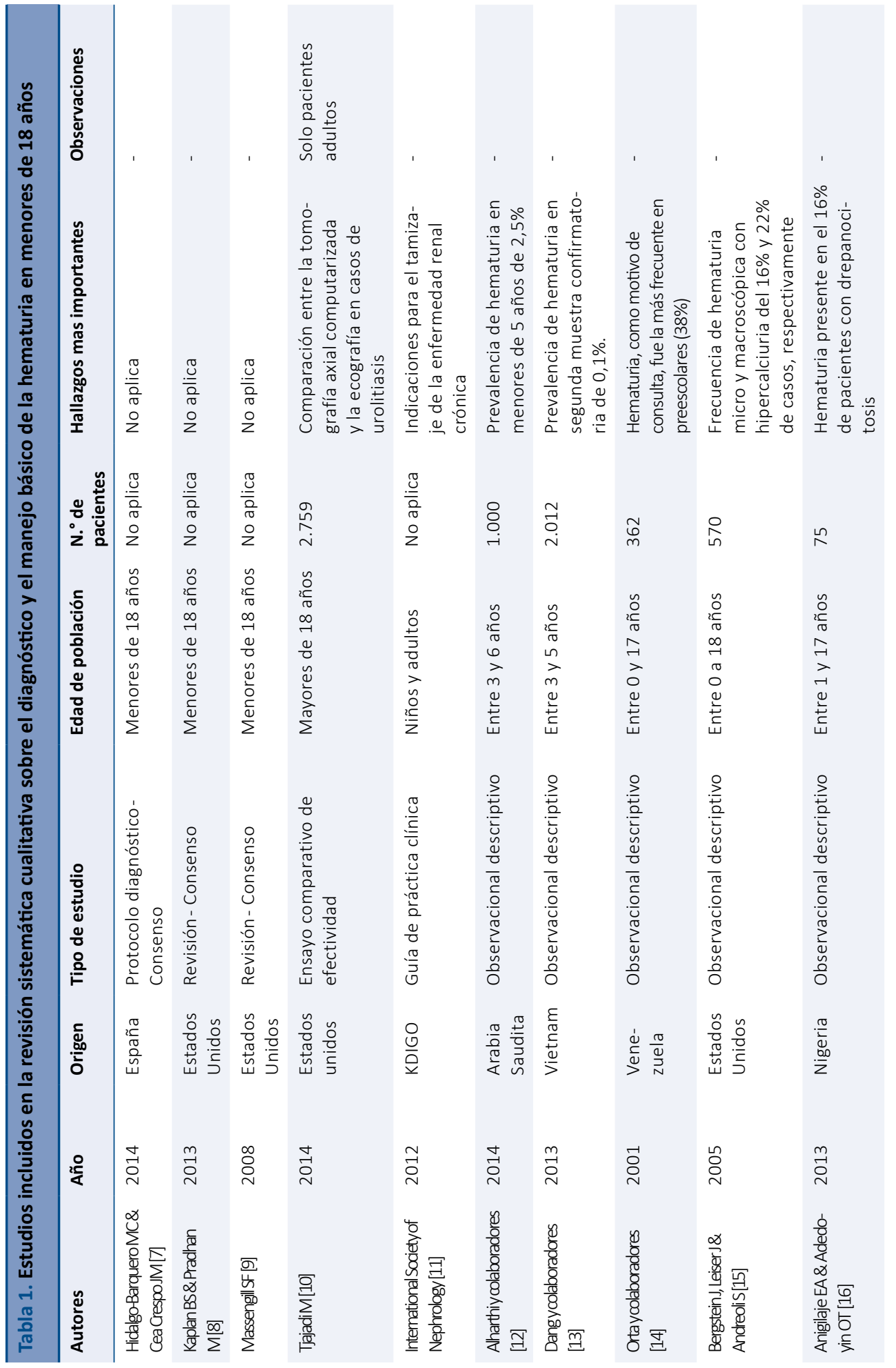




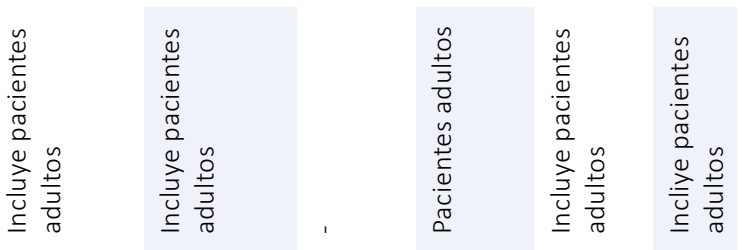

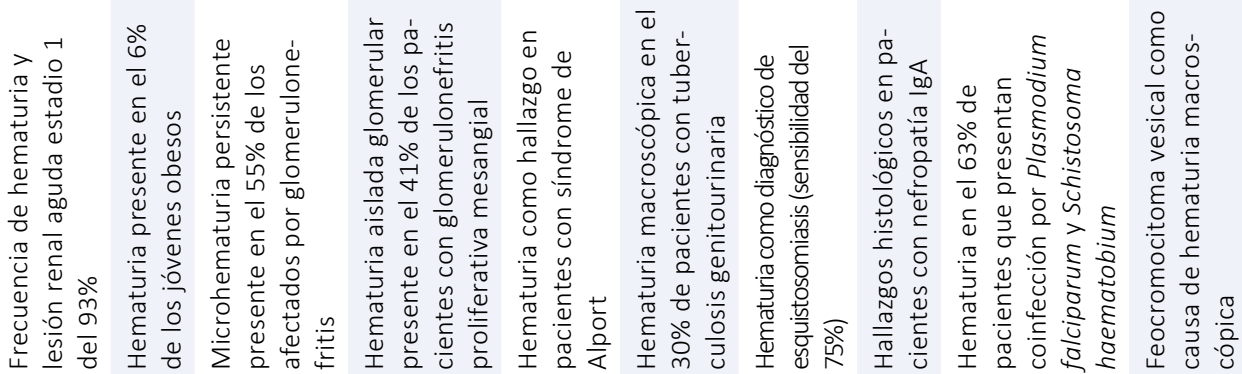

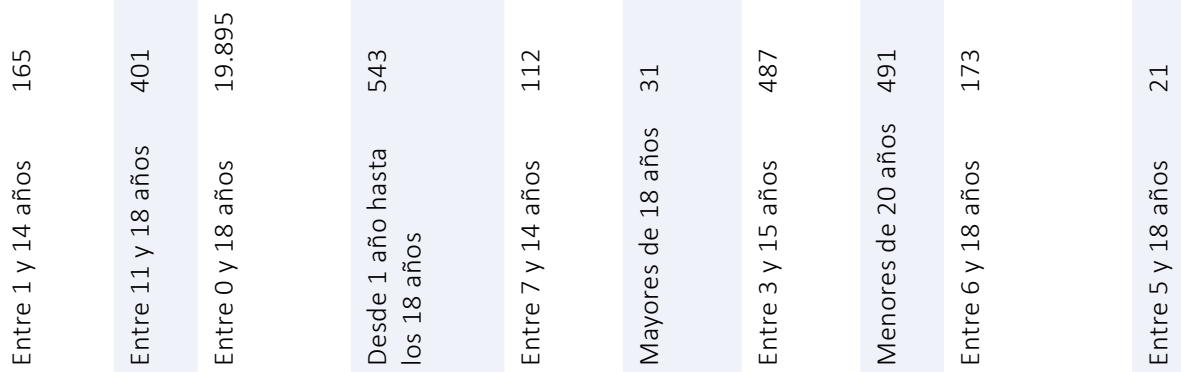
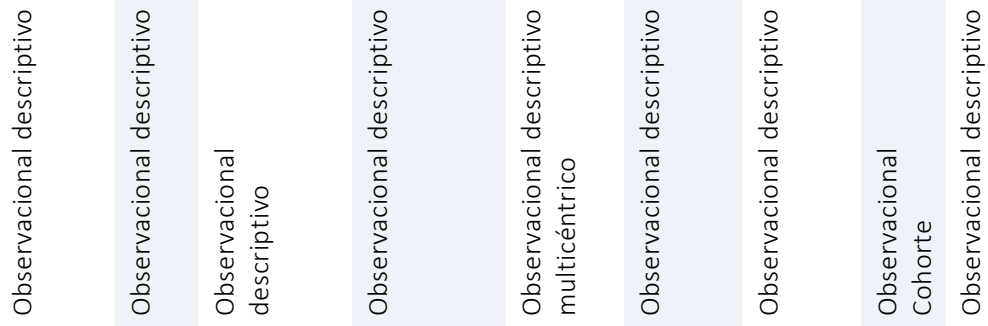

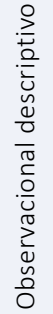

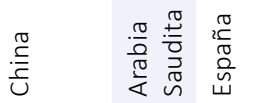

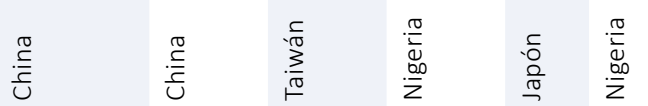

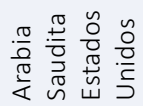

离

กั

离

$\stackrel{\sim}{\sim} \stackrel{0}{\stackrel{1}{\circ}}$

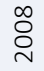

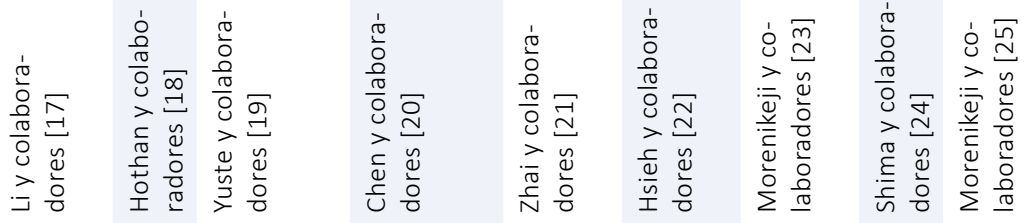

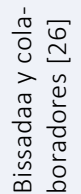


0
0
$\frac{0}{5}$
0
0
0
0
0
$\frac{0}{0}$
0
0
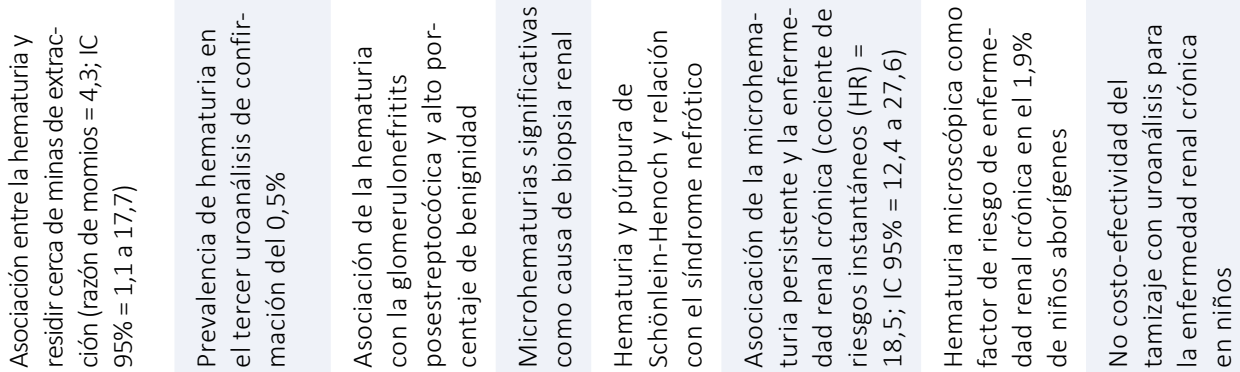

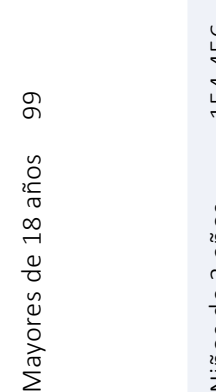
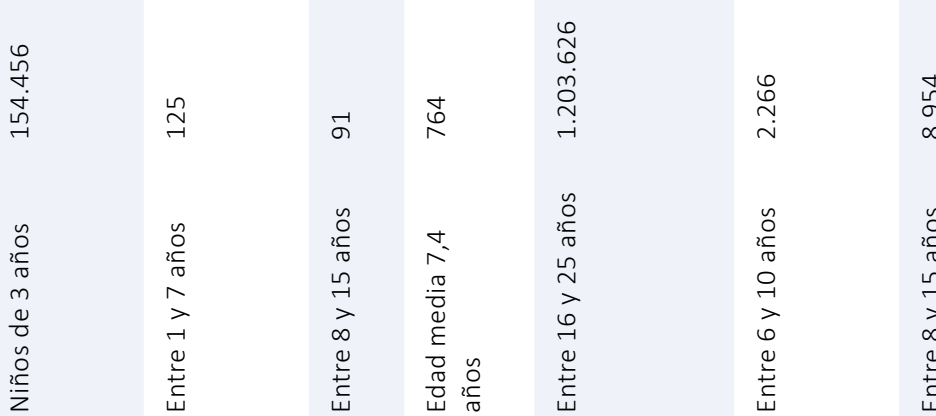

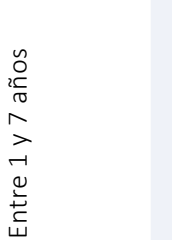
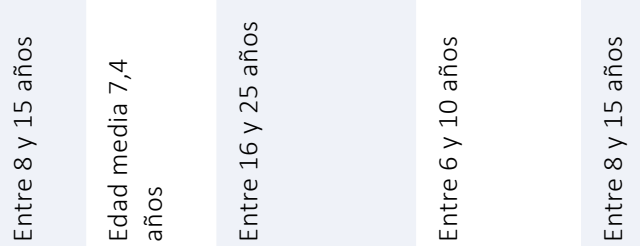

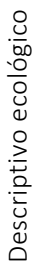

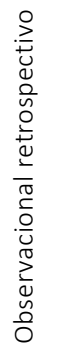

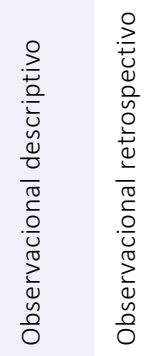

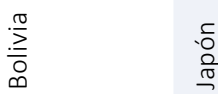

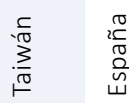

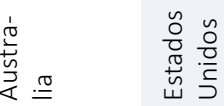

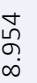

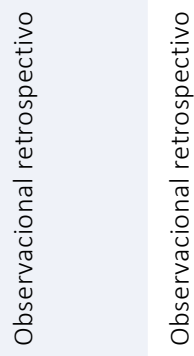

$\stackrel{?}{\geq}$

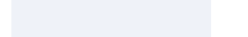

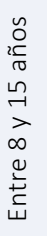

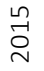

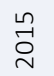

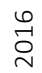

离

离

\&্ণ

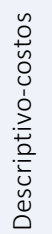

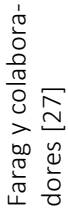

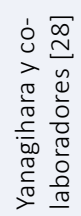

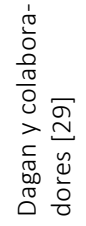

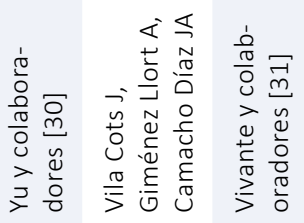

$\stackrel{0}{5}$
$\frac{5}{0}$
$\frac{0}{0}$

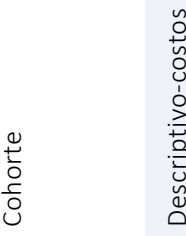

유 
de alto poder en orina fresca centrifugada o más de cinco eritrocitos por microlitro en orina fresca no centrifugada.

Hematuria microscópica persistente: si persiste la microhematuria aislada en tres o más muestras de orina tomadas con un intervalo de tiempo de dos a cuatro semanas.

Hematuria macroscópica: cuando la presencia de hematíes es lo suficientemente intensa para teñir la orina a simple vista (mayor a $1 \mathrm{~mL}$ de sangre por litro de orina; mayor a 5.000 hematíes por $\mu \mathrm{L}$ ). No obstante, a simple vista, puede ser confundida con el consumo de remolacha, colorantes de anilina, rifampicina, uratos de piridina o por cambios normales de la concentración de la orina que pueden producir la alteración de su coloración [1].

\section{Epidemiología}

La hematuria tiene una prevalencia mundial de al menos $1 \%$ al $2 \%$ en la población pediátrica en general, pero en estudios de campo alcanza el 2,5\% en los menores de cinco años [12]. En un estudio realizado en Vietnam, con más de 2.000 niños asintomáticos, con edades entre los cuatro y cinco años, provenientes de áreas remotas, a los que se les realizó uroanálisis con tira reactiva y confirmación en una segunda muestra, se encontró hematuria en el $0,1 \%$ de los participantes, con mayor número de niñas afectadas que niños [13].

En un estudio descriptivo realizado en Venezuela, en 2001, en población pediátrica que ingresó para consulta por nefrología pediátrica, se encontró hematuria en el $8,4 \%$, que representa en total el $1,1 \%$ de todas las consultas de dicho centro. El promedio de edad fue de 7,7+6,1 años, rango
0-17 años, $56 \%$ masculinos y $44 \%$ femeninos, $62 \%$ con hematuria microscópica y $38 \%$ con macro y microscópica. El grupo etario más afectado fue el de los preescolares $(34,3 \%)$, seguido por los escolares $(27,3 \%)$ [14]. Por su parte, en un estudio realizado en Cali-Colombia, sobre anomalías urinarias asintomáticas, se encontró que tenían una frecuencia del 7,9\% y entre las más prevalentes se encontraba la hematuria con un $14 \%$ de los casos [34].

\section{Enfermedades asociadas}

La hematuria está asociada a litiasis urinaria; hasta el $16 \%$ de los pacientes pediátricos con hipercalciuria tiene hematuria microscópica, y entre el $22 \%$ y el $43 \%$ tiene hematuria macroscópica $[15,35]$. Algunos de los niños que desarrollan hematuria tienen enfermedades graves como la anemia de células falciformes con desarrollo de nefropatía, así como lesión renal aguda en diversos estadios, con una prevalencia estimada del 93\% para el estadio 1 según estudios realizados en China [17]. Desafortunadamente, el tratamiento y el pronóstico dependen del lugar de residencia y de los recursos económicos con los que cuenta cada país [36].

La hematuria aislada asintomática en jóvenes está asociada a hipertensión, proteinuria y obesidad; esta ultima encontrada hasta en el $6 \%$ de los afectados [18].

\section{Causas}

En el estudio de hematuria es importante conocer si su origen es glomerular o extraglomerular (véase tabla 2) [9]. Por su parte, de acuerdo con su localización anatómica la hematuria puede ser glomerular, túbulo intersticial, del tracto urinario inferior o de origen vascular (véase tabla 3) [37]. 


\begin{tabular}{|c|c|c|}
\hline Características & $\begin{array}{l}\text { Hematuria } \\
\text { glomerular }\end{array}$ & $\begin{array}{l}\text { Hematuria } \\
\text { extraglomerular }\end{array}$ \\
\hline Color & $\begin{array}{l}\text { Oscuro o } \\
\text { Coca Cola }\end{array}$ & Rojo o rosa \\
\hline $\begin{array}{l}\text { Morfología } \\
\text { eritrocitos }\end{array}$ & Dismórfica & Normal \\
\hline Crenados & Hematíes & Ninguno \\
\hline Coágulos & Ausentes & Presentes \\
\hline Proteinuria & $>2$ cruces & $<2$ cruces \\
\hline \multicolumn{3}{|c|}{$\begin{array}{l}\text { Tomado y modificado de "Hematuria» por S. F. Massengill, } \\
\text { 2008, Pediatrics in Review, 29, p. 343. Copyright (C } 2008 \\
\text { por American Academy of Pediatrics [9]. }\end{array}$} \\
\hline
\end{tabular}

Tabla 3. Causas de hematuria segúnla localización anatómica

Glomerular

Glomerulonefritis posinfecciosa

Nefropatía por $\lg \mathrm{A}$

Hematuria benigna familiar

Púrpura de Schönlein-Henoch, nefritis lúpica

Ejercicio

Tubulo intersticial

Pielonefritis

Displasia quística renal

Enfermedad de células falciformes

Coagulopatía

Tuberculosis

Tracto urinario inferior

Urolitiasis

Hipercalciuria sin urolitiasis

Hiperoxaluria

Cistitis hemorrágica (viral o por medicamentos)

Uretritis bulbar idiopática

Tumores (angiomiolipoma, tumor de Wilms)

Esquistosomiasis

Vascular

Trombosis venosa renal

Síndrome de cascanueces

Tomado y modificado de «Haematuria and proteinuria in child hood» por A. Lunn and T. A.Forbes, 2012, Paediatr Child Heal (United Kingdom), 22, p. 316. (02012 por Elsevier Ltd. [37].
De acuerdo con un estudio realizado en España, en 19.895 pacientes con biopsia renal, a los que se les diagnosticó glomerulonefritis, la microhematuria persistente tuvo una incidencia del $55,1 \%$ y la hematuria macroscópica del 8,6\%, ambas altamente asociadas al hallazgo histológico de nefropatía por IgA y con una mayor frecuencia en los hombres $(64,7 \%)$ que en las mujeres $(62,4 \%)$ [19].

En otro estudio realizado en niños y adolescentes con hematuria aislada, definida como una hematuria glomerular, se encontró que el patrón histológico más frecuente fue la glomerulonefritis proliferativa mesangial con el $41,8 \%$ de los casos, seguido por la nefropatía por IgA con el 32,9\% [20]. Entre las posibilidades diagnósticas causales de hematuria también se deben considerar el síndrome de Alport, las mutaciones del colágeno tipo IV (p. ej la nefropatía por membrana basal delgada), la enfermedad renal poliquística autosómica dominante y la hiperoxaluria primaria [21,38-41].

Por su parte, la tuberculosis, endémica en los países en vías de desarrollo, en su forma renal, se puede encontrar en adolescentes y adultos con hematuria macroscópica, en el $30 \%$ de los pacientes $[22,42]$, e incluso se puede presentar en lactantes [43]. En cuanto a la esquistosomiasis, una parasitosis endémica en África con una prevalencia de alrededor del $57 \%$ en niños asintomáticos, es también una causa importante de microhematuria asintomática [23]. De la misma manera, la malaria por Plasmodium falciparum es otra causa importante de hematuria en los niños escolares de zonas rurales endémicas, con una prevalencia del $43,7 \%$, la cual aumenta al $63,8 \%$ cuando hay infección concomitantemente con Schistosoma haematobium [25]. 
La litiasis renal también es causa de hematuria, aunque es poco frecuente en niños, con una prevalencia estimada de $0,02 \%$ en poblaciones latinoamericanas [44]. En estos casos, cobra importancia el uroanálisis, ya que puede ser el primer indicio de hipercalciuria como precursora de la litiasis dado que es difícil demostrar las microlitiasis con imágenes obtenidas mediante urografía por tomografía computada [35]. Dependiendo de la composición de los cálculos, por ejemplo, ácido úrico, se relacionan con enfermedades como el síndrome de Fanconi, la enfermedad de células falciformes o la hiperuricosuria familiar [45].

El tumor de Wilms es la principal enfermedad maligna renal que se presenta con hematuria micro o macroscópica e indolora, con hipertensión y dolor abdominal, y que está acompañado, usualmente, de una masa renal palpable en los primeros cinco años de vida. La hematuria se puede observar en otros tumores menos frecuentes, como el feocromocitoma con hipertensión y el cáncer de vejiga, permitiendo su detección precoz a pesar de ser poco prevalente $[26,46]$. La trombosis renal también puede causar hematuria, dolor en el flanco y oliguria, y es más frecuente en la población neonatal [46]. Otra causa de hematuria en los niños podría estar dada por el consumo de agua contaminada con metales pesados como plomo, mercurio y cadmio, como se encontró en población adulta residente en lugares cercanos a minas de extracción intensiva en Bolivia [27].

\section{Evaluacion diagnóstica}

El paciente con microhematuria aislada no se debe estudiar con exámenes de extensión, como ecografías y tomografías, $u$ otros procedimientos invasivos, ya que en al menos el $25 \%$ de ellos el uroanálisis se normalizará en los siguientes cinco años. Por su parte, el paciente con microhematuria persistente requiere especial atención. La Academia Americana de Pediatría propone la siguiente estrategia, basada en completar tres pasos, la cual continúa siendo la más usada y de fácil aplicación [9].

\section{Primer paso}

Solicitar la evaluación de hematuria por tira reactiva en orina (sensibilidad: $73 \%$ al $89 \%$; especificidad: $81 \%$ al $93 \%$ ) [47], más microscopía óptica de alta resolución (sensibilidad: $52 \%$ al $73 \%$; especificidad: $98 \%$ al $100 \%$ ) [48] en muestra de orina de la mañana. Además, verificar el conteo de eritrocitos y el aspecto de la orina.

\section{Segundo paso}

Verificar si la hematuria es macroscópica o microscópica persistente. En caso de hematuria microscópica persistente verificar la morfología de los eritrocitos (eumórficos o dismórficos). Si hay eritrocitos dismórficos en más del 30\% y acantocitos en el 7\% o más se sospecha de una hematuria glomerular. Si este es el caso, además de realizar un análisis de los antecedentes familiares de glomerulopatías o sordera, se debe determinar la relación proteinuria/ creatinuria en orina esporádica, electrolitos séricos, nitrógeno ureico en sangre (BUN), creatinina sérica, los componentes 3 y 4 (C3 y C4) del complemento sérico, los anticuerpos antinucleares (ANAs) y los anticuerpos anticitoplasma de neutrófilos (ANCAs), para descartar o confirmar la presencia de una glomerulopatía tipo IgA , el síndrome de Alport, una vasculitis tipo púrpura de Schönlein-Henoch, un lupus 
eritematoso sistémico (LES) o una vasculitis pauciinmune. En caso de que los eritrocitos sean eumórficos se debe descartar la hipercalciuria, mediante la evaluación de la relación calciuria/creatinuria en orina esporádica, y reinterrogar al paciente sobre los antecedentes familiares de hematuria $[9,40]$.

\section{Tercer paso}

Si la hematuria es macroscópica se debe interrogar si hay antecedente de trauma directo o indirecto ( $p$. ej. en caso de tiempo prolongado en el uso de triciclos, bicicletas o brinca-brinca). El 95\% de estas situaciones se manejan de manera conservadora; se deben solicitar imágenes mediante ecografía, resonancia magnética o tomografía, según la sospecha clínica y la condición del paciente, teniendo en cuenta que el $5 \%$ de los pacientes pueden tener anormalidades renales, como riñones ectópicos pélvicos, riñones en herradura e hidronefrosis por estrechez pieloureteral, las cuales son descubiertas ante un trauma leve o con el aumento de la edad. Todas estas alteraciones ameritan estudios de imágenes y funcionales antes de decidir el requerimiento quirúrgico cuando sea necesario $[49,50]$. Es importante tener siempre precaución con los medios de contraste antes de solicitarlos.

Si la hematuria es macroscópica siempre se debe pensar en hipercalciuria en todas las edades, debido a su alta prevalencia, especialmente en preescolares y escolares. En caso de que sea dolorosa, se deben descartar los cálculos en las vías urinarias con una imagen, de preferencia obtenida mediante urografía por tomografía computada sin medio de contraste, previa evaluación de la función renal. No obstante, esto aún es motivo de debate puesto que estudios en adultos muestran que la ecografía renal como primer estudio, respecto a la tomografía, tiene igual especificidad y evita la exposición a la radiación [10].

Por su parte, si la hematuria macroscópica es indolora se indica evaluar la relación proteinuria/creatinuria en orina esporádica para determinar una posible vasculitis tipo púrpura de Schönlein-Henoch, puesto que está relacionada con los síndromes nefróticos en su presentación [51].

En la figura 2 se presenta el algoritmo propuesto para el diagnóstico de la hematuria en niños.

\section{Tratamiento y pronóstico}

El tratamiento y pronóstico de la hematuria depende de la causa desencadenante del proceso primario. El pronóstico de la hematuria en la niñez es benigno en un alto porcentaje, sobre todo en los preescolares, en los cuales se ha demostrado que presentan hematuria en el primer examen de orina en el $8,2 \%$ de los casos y en el tercer uroanálisis de confirmación esta prevalencia disminuye al 0,5\% [28]. En otras poblaciones el porcentaje de hematuria significativa en la primera muestra ha sido tan solo del $0,8 \%$ [52]. No obstante, el paciente pediátrico con hematuria sintomática y presencia de signos de alarma como hipertensión, proteinuria y pérdida de peso, debe ser manejado por nefrología pediátrica.

En caso de masas o cálculos de gran tamaño se debe realizar un enfoque quirúrgico en cabeza del urólogo pediatra [50]. No se debe olvidar que el cáncer genitourinario está presente en algunos niños con hematuria, como es el caso del cáncer de vejiga y 


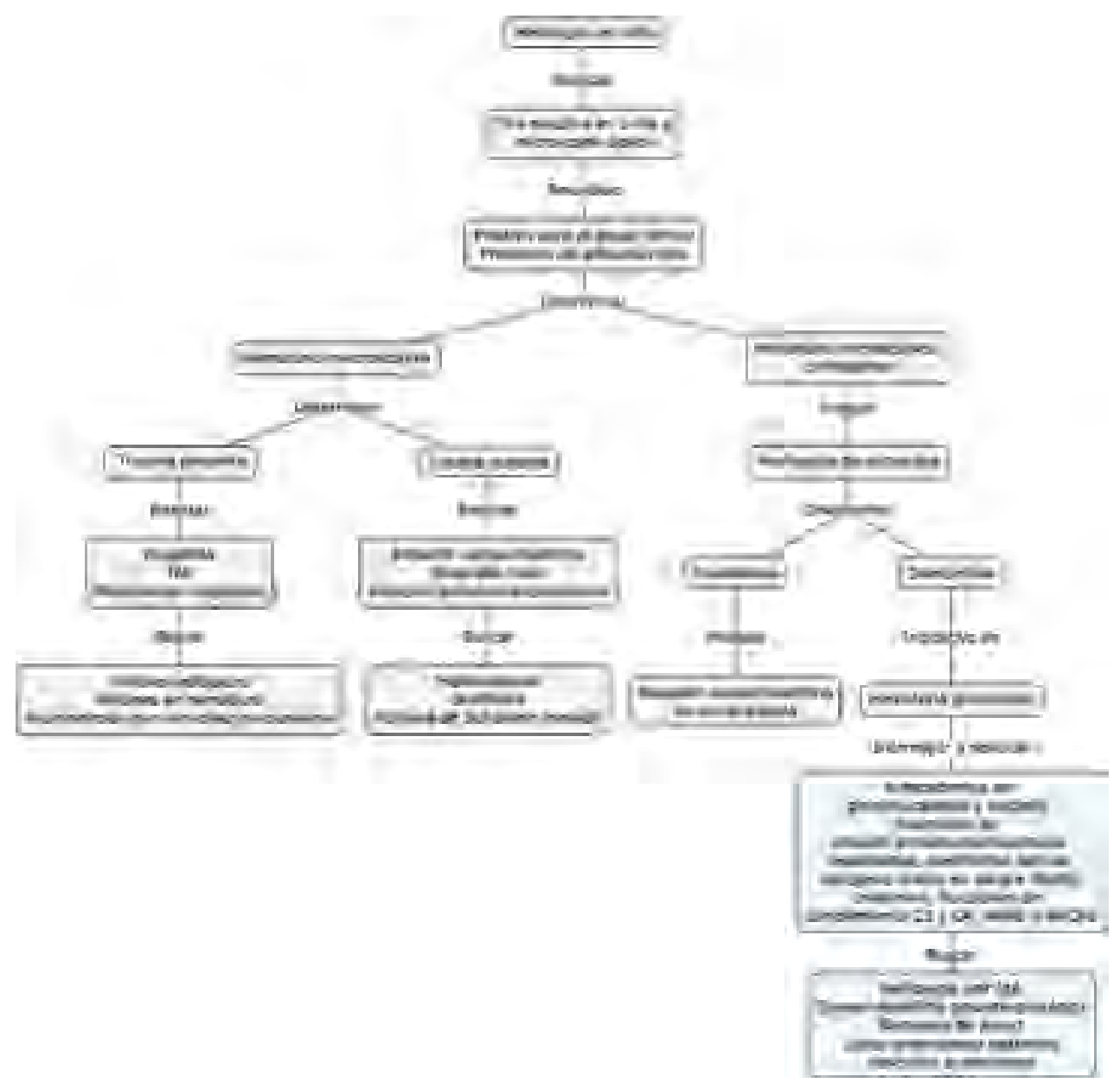

Figura 2. Algoritmo diagnóstico de la hematuria en niños. Tomado y modificado de "Hematuria" por S. F. Massengill, 2008, Pediatr Rev, 29, p. 344. Copyright ${ }^{\circledR} 2008$ por The American Academy of Pediatrics. $\mathrm{TAC}=$ tomografía axial computarizada, ANAs = anticuerpos antinucleares, ANCAs= anticuerpos anticitoplasma de neutrófilos.

el tumor de Wilms, y, por lo tanto, ante el hallazgo de una masa abdominal se deben tomar las respectivas precauciones [53].

Los pacientes que sufren hematuria de etiología posinfecciosa, como la glomerulonefritis posestreptocócica, usualmente entre las edades de 5 a 12 años, tienen un buen pronóstico. En un estudio de seguimiento de niños que habían sufrido glome- rulonefritis posinfecciosa se encontró que 48 meses después 100 de 125 pacientes tenían una función renal normal, cinco tenían hipertensión y solo uno proteinuria persistente [29].

Dado que la glomerulonefritis posestreptocócica generalmente es benigna en nuestro medio, siempre se debe diferenciar de la glomerulonefritis por agentes diferen- 
tes, entre ellos los virus, en la cual no se agota el componente 3 (C3) del complemento, como sí sucede en la glomerulonefritis posestreptocócica. En los casos de no consumo del complemento y presencia de hematuria microscópica persistente se debe descartar durante el seguimiento una nefropatía por IgA, la cual es la glomerulopatía mas frecuente, con un pronóstico diferente y que requiere para su diagnóstico una biopsia renal [24].

Otras indicaciones de biopsia renal para el estudio de hematuria microscópica clínicamente significativa son las microhematurias asociadas con proteinuria significativa entre $100 \mathrm{mg} / 24$ horas y $500 \mathrm{mg} / 24$ horas, el síndrome nefrótico corticodependiente y corticorresistente, y la falla renal inexplicada, entre otras [30].

En un estudio de cohorte retrospectiva, realizado en Israel, en 1.203.626 jóvenes entre 16 y 25 años seguidos por más de 20 años, se encontró que de 3.690 de ellos, que fueron diagnósticados con hematuria microscópica persistente (1.199.936 fueron negativos para esta condición), 26 $(0,7 \%)$ presentaron progresión a enfermedad renal en etapa terminal (incidencia = 34,0/100.000 personas-año; intervalo de confianza del $95 \%=22,2$ a 49,9 y cociente de riesgos (respecto a pacientes sin hematuria previa) $=18,5$; intervalo de confianza del $95 \%=12,4$ a 27,6$)[31]$.

Otro estudio de cohorte prospectiva de 2.266 niños aborígenes y no aborígenes de Australia, con 1.506 de ellos seguidos durante cuatro años, demostró una persistencia de la hematuria microscópica, como factor de riesgo de enfermedad renal crónica, en el 1,9\% de ellos, sin mayor diferencia en la prevalencia de esta al discriminar entre los niños aborígenes $(1,8 \%)$ y los no aborígenes (2,0\%) [32].

Si bien la Academia Americana de Pediatría, desde el 2007, no aconseja el tamizaje universal de la enfermedad renal crónica con tira reactiva en muestra aislada de orina, teniendo en cuenta el parámetro de hematuria o proteinuria, debido a que su costoefectividad como método de tamizaje es limitada [33], es preciso que se realice en pacientes de alto riesgo como los niños obesos, hipertensos, diabéticos o con antecedentes de enfermedad renal crónica hereditaria, de acuerdo con lo indicado en las guías del consorcio KDIGO (del inglés, Kidney Disease: Improving Global Outcomes) del 2012 [11].

\section{Conclusiones}

La hematuria en pediatría es un trastorno relativamente común que se presenta en un $7 \%$ al $8 \%$ de la población. Dado que es un signo de alarma, el médico general y el pediatra de atención primaria deben estar atentos a su presencia para darle un manejo integral al niño, lo cual requiere que, desde un principio, se apliquen los conceptos básicos sobre su enfoque inicial, diagnóstico y tratamiento, que evite la realización de procedimientos innecesarios en los pacientes con estos hallazgos.

\section{Bibliografía}

1. Meyers KE. Evaluation of hematuria in children. Urol Clin North Am 2004; 31: 559-573.

2. Chung H-M, Liao Y-M, Tsai Y-C, Liu M-C. Microscopic hematuria in children. Urol Sci 2011; 22: 93-96.

3. Moghtaderi M, Noohi AH, Safaeyan B, Abbasi A, Sabsechian M, Meherkash M. Screening for microscopic hematuria in school-age children of Gorgan City. Iran J Kidney Dis 2014; 8: 70-72.

4. Lambert SM. Pediatric Urological Emergencies. Pediatr Clin 2012; 59: 965-976. 
5. Cho BS, Hahn WH, Cheong HI, Lim I, Ko CW, Kim SY, et al. A nationwide study of mass urine screening tests on Korean school children and implications for chronic kidney disease management. Clin Exp Nephrol 2013; 17: 205-210.

6. Lopera-Medina MM. La enfermedad renal crónica en Colombia: necesidades en salud y respuesta del Sistema General de Seguridad Social en Salud. Gerenc Polít Salud 2016; 15: 212-233.

7. Hidalgo-Barquero MC, Cea-Crespo JM. Hematuria. Protoc diagn ter pediat 2014; 1: 53-68.

8. Kaplan BS, Pradhan M. Urinalysis interpretation for pediatricians. Pediatr Ann 2013; 42: 45-51.

9. Massengill SF. Hematuria. Pediatr Rev 2008; 29: 342348.

10. Tjajadi M. Ultrasonography Versus Computed Tomography for Suspected Nephrolithiasis. J Emerg Med 2014; 47: 752-753.

11. International Society of Nephrology. KDIGO 2012 Clinical Practice Guideline for the Evaluation and Management of Chronic Kidney Disease. Kidney Int Suppl 2013; 3: 1-150.

12. Alharthi AA, Taha AA, Edrees AE, Elnawawy AN, Abdelrahman AH. Screening for urine abnormalities among preschool children in western Saudi Arabia. Saudi Med J 2014; 35: 1477-1481.

13. Dang LNN, Doan TLB, Doan NH, Pham TKH, Smets F, Thi MHT, et al. Epidemiological urinalysis of children from kindergartens of Can Gio, Ho Chi Minh City - Vietnam. BMC Pediatr 2013; 13: 183.

14. Orta N, Sanna V, Moriyón JC, de Orta S, Domínguez L, Zibaoui $\mathrm{P}$, et al. Hematuria en niños: analysis of aetiology and characteristics in a national reference centre, Valencia, Venezuela. Rev Chil Pediatr 2001; 72: 92-99.

15. Bergstein J, Leiser J, Andreoli S. The clinical significance of asymptomatic gross and microscopic hematuria in children. Arch Pediatr Adolesc Med 2005; 159: 353-355.

16. Anigilaje EA, Adedoyin OT. Correlation between dipstick urinalysis and urine sediment microscopy in detecting haematuria among children with sickle cell anaemia in steady state in Ilorin, Nigeria. Pan Afr Med J 2013; 15: 1-7.

17. Li Z, Kang Z, Duan C, Wu T, Zhang L, Xun M, et al. Clinical and pathological features of acute kidney injury in children. Ren Fail 2014; 36: 1023-1028.

18. Hothan KA, Alasmari BA, Alkhelaiwi OK, Althagafi KM, Alkhaldi AA, Alfityani AK, et al. Prevalence of hypertension, obesity, hematuria and proteinuria amongst healthy adolescents living in Western Saudi Arabia. Saudi Med J 2016; 37: 1120-1126.

19. Yuste C, Rivera F, Moreno JA, López-Gómez JM. Haematuria on the Spanish Registry of Glomerulonephritis. Sci Rep 2016; 6: 19732.

20. Chen GL, Zhu J, Song MH, Ma L, Pan T, Yang Q, et al. Clinicopathologic analysis of isolated hematuria in child/ adolescent and adult patients. Pol J Pathol 2015; 66: 353360.

21. Zhai $Y, X u H$, Shen Q, Cao Q, Zhu G, Wei M, et al. Renal histological features of school-age children with asymptomatic haematuria and/or proteinuria: a multicenter study. Nephrology (Carlton) 2014; 19: 426-431.

22. Hsieh HC, Lu PL, Chen YH, Chen TC, Tsai JJ, Chang K, et al. Genitourinary tuberculosis in a medical center in southern Taiwan: an eleven-year experience. J Microbiol Immunol Infect 2006; 39: 408-413.

23. Morenikeji O, Quazim J, Omoregie C, Hassan A, Nwuba $\mathbf{R}$, Anumudu $\mathbf{C}$, et al. A cross-sectional study on urogenital schistosomiasis in children; haematuria and proteinuria as diagnostic indicators in an endemic rural area of Nigeria. Afr Health Sci 2014; 14: 390-396.

24. Shima $\mathrm{Y}$, Nakanishi $\mathrm{K}$, Hama T, Sato M, Mukaiyama H, Togawa $\mathrm{H}$, et al. Biopsy timing and Oxford classification variables in childhood/adolescent IgA nephropathy. Pediatr Nephrol 2015; 30: 293-299.

25. Morenikeji OA, Eleng IE, Atanda OS, Oyeyemi OT. Renal related disorders in concomitant Schistosoma haematobium-Plasmodium falciparum infection among children in a rural community of Nigeria. J Infect Public Health 2016; 9: 136-142.

26. Bissada NK, Safwat AS, Seyam RM, Al Sobhi S, Hanash KA, Jackson RJ, et al. Pheochromocytoma in children and adolescents: a clinical spectrum. J Pediatr Surg 2008; 43: 540-543.

27. Farag S, Das R, Strosnider WH, Wilson RT. Possible health effects of living in proximity to mining sites near Potosi, Bolivia. J Occup Environ Med 2015; 57: 543-551.

28. Yanagihara $T$, Hamada $R$, Ishikura $K$, Uemura $O$, Matsuyama T, Takahashi S, et al. Urinary screening and urinary abnormalities in 3-year-old children in Japan. Pediatr Int 2015; 57: 354-358.

29. Dagan R, Cleper R, Davidovits M, Sinai-Trieman L, Krause I. Post-Infectious Glomerulonephritis in Pediatric Patients over Two Decades: Severity-Associated Features. Isr Med Assoc J 2016; 18: 336-340.

30. Yu M-C, Lee F, Huang W-H, Hsueh S. Percutaneous ultrasound-guided renal biopsy in children: The need for renal biopsy in pediatric patients with persistent asymptomatic microscopic hematuria. Biomed J 2014; 37: 391-397.

31. Vivante A, Afek A, Frenkel-Nir Y, Tzur D, Farfel A, Golan E, et al. Persistent asymptomatic isolated microscopic hematuria in Israeli adolescents and young adults and risk for end-stage renal disease. JAMA 2011; 306: 729736.

32. Haysom L, Williams R, Hodson EM, Lopez-Vargas PA, Roy LP, Lyle DM, et al. Natural history of chronic kidney disease in Australian Indigenous and non-Indigenous children: a 4-year population-based follow-up study. Med J Aust 2009; 190: 303-306.

33. Sekhar DL, Wang L, Hollenbeak CS, Widome MD, Paul IM. A cost-effectiveness analysis of screening urine dipsticks in well-child care. Pediatrics 2010; 125.

34. Restrepo de Rovetto C. Hematuria en la niñez. CCAP 2002; 2: 30-40.

35. Marzuillo P, Guarino S, Apicella A, La Manna A, Polito C. Why we need a higher suspicion index of urolithiasis in children. J Pediatr Urol 2017; 13: 164-171.

36. Ingelfinger JR, Kalantar-Zadeh K, Schaefer F. Averting the legacy of kidney disease--focus on childhood. Kidney Int 2016; 89: 512-518.

37. Lunn A, Forbes TA. Haematuria and proteinuria in childhood. Paediatr Child Heal (United Kingdom) 2012; 22: 315-321.

38. Feng CY, Xia YH, Wang WJ, Xia J, Fu HD, Wang X, et al. Persistent asymptomatic isolated hematuria in children: 
clinical and histopathological features and prognosis. World J Pediatr 2013; 9: 163-168.

39. Chan MM, Gale DP. Isolated microscopic haematuria of glomerular origin: clinical significance and diagnosis in the 21st century. Clin Med (Lond) 2015; 15: 576-580.

40. Feld LG, Waz WR, Perez LM, Joseph DB. Hematuria. An integrated medical and surgical approach. Pediatr Clin North Am 1997; 44: 1191-1210.

41. Jiang D, Geng H. Primary Hyperoxaluria. New Engl J Med 2017; 376: e33.

42. Cruz AT, Starke JR. Clinical manifestations of tuberculosis in children. Paediatr Respir Rev 2007; 8: 107-117.

43. Mayans AF, Feu OA, Til HA, Terroba YA, Fernández RB, Tur AB, et al. Renal Tuberculosis in a 9 Months Old: Case Report and Review of the Literature. Urol Case Rep 2017; 13: 104-106.

44. Velásquez-Forero F, Esparza M, Salas A, Medeiros M Toussaint G, Llach F. Risk factors evaluation for urolithiasis among children. Bol Med Hosp Infant Mex 2016; 73 228-236.

45. Abou-Elela A. Epidemiology, pathophysiology, and man- agement of uric acid urolithiasis: A narrative review. J Adv Res 2017; 8: 513-527.

46. Goldenberg NA, Bernard TJ. Venous thromboembolism in children. Pediatr Clin North Am 2008; 55: 305-322, vii.

47. Utsch B, Klaus G. Urinalysis in children and adolescents. Dtsch Arztebl Int 2014; 111: 617-625; quiz 626.

48. Cohen RA, Brown RS. Clinical practice. Microscopic hematuria. N Engl J Med 2003; 348: 2330-2338.

49. Sinha CK, Lander A. Trauma in children: abdomen and thorax. Surg 2013; 31: 123-129.

50. Tanaka ST, Brock JW, 3rd. Pediatric urologic conditions, including urinary infections. Med Clin North Am 2011; 95: 1-13.

51. Vila Cots J, Giménez Llort A, Camacho Díaz JA, Vila Santandreu A. Nefropatía en la púrpura de SchönleinHenoch: estudio retrospectivo de los últimos 25 años. An Pediatr (Barc) 2007; 66: 290-293.

52. Cho BS, Kim SD. School urinalysis screening in Korea. Nephrology (Carlton) 2007; 12.

53. Raab CP, Gartner JC, Jr. Diagnosis of childhood cancer. Prim Care 2009; 36: 671-684.

Introduction: Isolated hematuria in children is a frequent finding in the daily clinical practice of the general physician and pediatrician, being a diagnostic challenge to find out its etiology and management. Objective: To describe the basic guidelines for the diagnosis and management of isolated hematuria in children under 18 years of age by the general physician and the pediatrician. Materials and methods: A systematic bibliographic search was carried out in the databases PubMed, ScienceDirect, LILACS and Embase, using key words from DeCS (Spanish) and MeSH (English) through combinations with the conjunction "AND" or the disjunction "OR ", of manuscripts such as observational studies, clinical trials, clinical practice guidelines and systematic reviews, published between 1995 and 2017 that described the basic diagnosis and management of hematuria. The quality of the articles was evaluated through the PRISMA, CONSORT and STROBE instruments, as was appropriate. Results: 402 publications were identified, of which 34 met the criteria and 28 were selected to carry out this review. Conclusions: Isolated hematuria is defined by the finding of five erythrocytes per high power field in centrifuged fresh urine or more than five erythrocytes per microliter in fresh non-centrifuged urine Diagnosis and treatment are carried out in steps: a) clinical history, and b) confirmation by high resolution optical microscopy. Differentiating benign hematuria from those requiring paraclinical extension is essential to confirm it earlier, avoid invasive procedures, decrease health expenditure and make predictive follow-up.

Key words: hematuria, children, diagnosis, systematic review. 